\title{
DNS of mass transfer from bubbles rising in a vertical channel $^{\star}$
}

\author{
Néstor Balcázar-Arciniega ${ }^{2,1[0000-0003-0776-2086]}$, Joaquim \\ Rigola $^{1[0000-0002-6685-3677]}$, and Assensi Oliva1[0000-0002-2805-4794] \\ 1 Heat and Mass Transfer Technological Center (CTTC), Universitat Politècnica de \\ Catalunya-BarcelonaTech (UPC). ETSEIAT, Colom 11, 08222, Barcelona, Spain \\ nestorbalcazardyahoo.es, \{nestor, cttc\}@ettc.upc.edu \\ http://www.cttc.upc.edu/ \\ 2 Termo Fluids S.L., Avda Jacquard 97 1-E, 08222, Terrassa (Barcelona), Spain \\ http://www.termofluids.com/
}

\begin{abstract}
This work presents Direct Numerical Simulation of mass transfer from buoyancy-driven bubbles rising in a wall-confined vertical channel, through a multiple markers level-set method. The Navier-Stokes equations and mass transfer equation are discretized using a finite volume method on a collocated unstructured mesh, whereas a multiple markers approach is used to avoid the numerical coalescence of bubbles. This approach is based on a mass conservative level-set method. Furthermore, unstructured flux-limiter schemes are used to discretize the convective term of momentum equation, level-set advection equations, and mass transfer equation, to improve the stability of the solver in bubbly flows with high Reynolds number and high-density ratio. The level-set model is used to research the effect of bubble-bubble and bubble-wall interactions on the mass transfer from a bubble swarm rising in a vertical channel with a circular cross-section.
\end{abstract}

Keywords: Mass transfer - Bubbly flow $\cdot$ Vertical channel $\cdot$ Flux-limiters $\cdot$ Unstructured meshes $\cdot$ Level-set method $\cdot$ Finite volume method $\cdot$ High-Performance Computing.

\section{Introduction}

Mass transfer in bubbly flows is a ubiquitous phenomenon in natural and industrial applications. For example, bubble columns are used in chemical engineering to promote chemical reactions, as well as to improve heat and mass transfer rates. Therefore, understanding this phenomenon has both practical and scientific motivations. As

\footnotetext{
* This work has been financially supported by the Ministerio de Economía y Competitividad, Secretaría de Estado de Investigación, Desarrollo e Innovación (MINECO), Spain (ENE201570672-P), and by Termo Fluids S.L. Néstor Balcázar acknowledges financial support of the Programa Torres Quevedo, Ministerio de Economía y Competitividad, Secretaría de Estado de Investigación, Desarrollo e Innovación (PTQ-14-07186), Spain. Three-dimensional simulations were carried out using computing time awarded by PRACE 14th-Call (project 2016153612), and RES (project $F I-2018-1$ - 0025), on the supercomputer MareNostrum IV based in Barcelona, Spain. Computational resources provided by RES (project $F I-2018-3-0037)$ on the supercomputer Altamira, supported by Santander Supercomputing group at the University of Cantabria (IFCA-CSIC), are acknowledged.
}

ICCS Camera Ready Version 2019

To cite this paper please use the final published version:

DOI $10.1007 / 978-3-030-22747-0 \_45$ 
a complement to theoretical and experimental approaches, the development of supercomputers has promoted High-Performance computing (HPC) and Direct Numerical Simulation (DNS) of Navier-Stokes equations, as another methodology to design noninvasive and controlled numerical experiments of bubbly flows. Indeed, during the last decades multiple numerical methods have been introduced for DNS of two-phase flows: volume-of-fluid (VOF) methods [26], level-set (LS) methods [33|36], conservative level-set (CLS) methods [32]4], front tracking (FT) methods [42], and hybrid VOF/LS methods [37|39]7]. Furthermore, some of these numerical approaches have been extended to include heat transfer or mass transfer phenomenon in two-phase flows [21|14]3 15 20[12]. On the other hand, few works have reported DNS of mass transfer in bubble swarms [2]35|29|12]. Although previous publications have researched mass transfer from bubbles rising on unconfined domains by using VOF, LS, VOF/LS, and FT methods, there are no previous studies in the context of wall-confined vertical columns and CLS method. Therefore, this work aims to present a numerical study of mass transfer from bubbles rising in a vertical pipe, in the framework of a multiple-marker CLS methodology introduced by [5|9|12]. As further advantages, the CLS method [4]12] was designed for three-dimensional collocated unstructured meshes, whereas the accumulation of mass conservation error inherent to standard level-set methods is circumvented. Moreover, unstructured flux-limiters schemes as first introduced in [4]8[12], are used to discretize convective terms of transport equations, in order to avoid numerical oscillations around discontinuities and to minimize the so-called numerical diffusion. This numerical approach has demonstrated to improve the numerical stability of the unstructured multiphase solver [4[5]6/7/8/12] for bubbly flows with high Reynolds number and high-density ratio.

This paper is organized as follows: The mathematical model and numerical methods are reviewed in section 2 Numerical experiments are presented in section 3 . Concluding remarks and future work are discussed in section 4

\section{Mathematical model and numerical methods}

\subsection{Incompressible two-phase flow}

The Navier-Stokes equations for the dispersed fluid $\left(\Omega_{d}\right)$ and continuous fluid $\left(\Omega_{c}\right)$ are introduced in the framework of the so-called one-fluid formulation [42], which includes a singular source term for the surface tension force at the interface $\Gamma$ [42|4|12]:

$$
\begin{gathered}
\frac{\partial}{\partial t}(\rho \mathbf{v})+\nabla \cdot(\rho \mathbf{v v})=-\nabla p+\nabla \cdot \mu(\nabla \mathbf{v})+\nabla \cdot \mu(\nabla \mathbf{v})^{T}+\left(\rho-\rho_{0}\right) \mathbf{g}+\mathbf{f}_{\sigma}, \\
\nabla \cdot \mathbf{v}=0,
\end{gathered}
$$

where $\mathbf{v}$ is the fluid velocity, $p$ denotes the pressure field, $\mathbf{g}$ is the gravitational acceleration, $\rho$ is the fluid density, $\mu$ is the dynamic viscosity, $\mathbf{f}_{\sigma}$ is the surface tension force per unit volume concentrated at the interface, subscripts $d$ and $c$ denote the dispersed phase and continuous phase respectively. Physical properties are constant at each fluid-phase with a jump discontinuity at $\Gamma$ :

$$
\rho=\rho_{d} H_{d}+\rho_{c} H_{c}, \mu=\mu_{d} H_{d}+\mu_{c} H_{c} .
$$

ICCS Camera Ready Version 2019

To cite this paper please use the final published version:

$\begin{array}{lll}\text { DOI } & 10.1007 / 978-3-030-22747-0 \_45\end{array}$ 
Here $H_{c}$ is the Heaviside step function that is one at fluid $c\left(\Omega_{c}\right)$ and zero elsewhere, whereas $H_{d}=1-H_{c}$. At discretized level a continuous treatment of physical properties is adopted in order to avoid numerical instabilities around $\Gamma$. The force $-\rho_{0} \mathbf{g}$ included in Eq. (1), with $\rho_{0}=V_{\Omega}^{-1} \int_{\Omega}\left(\rho_{d} H_{d}+\rho_{c} H_{c}\right) d V$, avoids the acceleration of the flow field in the downward vertical direction, when periodic boundary conditions are applied on the $y$-axis (aligned to $\mathbf{g}$ ) [22[5]9]12].

\subsection{Multiple marker level-set method and surface tension}

The conservative level-set method (CLS) introduced by [4]8[12] for interface capturing on three-dimensional unstructured meshes, is used in this work. Furthermore, the multiple markers approach [195] as introduced in [5|8|12] for the CLS method, is employed to avoid the so-called numerical coalescence inherent to standard interface capturing methods. In this context, each bubble is represented by a CLS function [5]8|9]12], whereas the interface of the ith fluid particle is defined as the 0.5 iso-surface of the CLS function $\phi_{i}$, with $i=1,2, \ldots, n_{d}$ and $n_{d}$ defined as the total number of bubbles in $\Omega_{d}$. Since the incompressibility constraint (Eq. 2), the ith interface transport equation can be written in conservative form as follows:

$$
\frac{\partial \phi_{i}}{\partial t}+\nabla \cdot \phi_{i} \mathbf{v}=0, i=1, . ., n_{d} .
$$

Furthermore, a re-initialization equation is introduced to keep a sharp and constant CLS profile on the interface:

$$
\frac{\partial \phi_{i}}{\partial \tau}+\nabla \cdot \phi_{i}\left(1-\phi_{i}\right) \mathbf{n}_{i}^{0}=\nabla \cdot \varepsilon \nabla \phi_{i}, i=1, . ., n_{d} .
$$

where $\mathbf{n}_{i}^{0}$ denotes $\mathbf{n}_{i}$ at $\tau=0$. This equation is advanced in pseudo-time $\tau$ up to achieve the steady state. It consists of a compressive term, $\phi_{i}\left(1-\phi_{i}\right) \mathbf{n}_{i}^{0}$, which forces the CLS function to be compressed onto the interface along the normal vector $\mathbf{n}_{i}$. Furthermore, the diffusive term, $\nabla \cdot \varepsilon \nabla \phi_{i}$, keeps the level-set profiles with characteristic thickness $\varepsilon=0.5 h^{0.9}$, where $h$ is the grid-size [4]8|12]. Geometrical information at the interface, such as normal vectors $\mathbf{n}_{i}$ and curvatures $\kappa_{i}$, are computed from the CLS function:

$$
\mathbf{n}_{i}\left(\phi_{i}\right)=\frac{\nabla \phi_{i}}{\left\|\nabla \phi_{i}\right\|}, \kappa_{i}\left(\phi_{i}\right)=-\nabla \cdot \mathbf{n}_{i}, i=1, . ., n_{d} .
$$

Surface tension forces are calculated by the continuous surface force model [16], extended to the multiple marker CLS method in [5 8|9|12]:

$$
\mathbf{f}_{\sigma}=\sum_{i=1}^{n_{d}} \sigma \kappa_{i}\left(\phi_{i}\right) \nabla \phi_{i}
$$

where $\sigma$ is the surface tension coefficient. Finally, in order to avoid numerical instabilities at the interface, fluid properties in Eq. (3) are regularized by using a global level-set function $\phi[5[8 \mid 12]$, defined as follows:

$$
\phi=\min \left\{\phi_{1}, \ldots, \phi_{n_{d}}\right\} .
$$

ICCS Camera Ready Version 2019

To cite this paper please use the final published version:

$\begin{array}{lll}\text { DOI } & 10.1007 / 978-3-030-22747-0 \_45\end{array}$ 
Thus, Heaviside functions presented in Eq.(3) are regularized as $H_{d}=1-\phi$ and $H_{c}=\phi$. In this work $0<\phi \leq 0.5$ for $\Omega_{d}$, and $0.5<\phi \leq 1$ for $\Omega_{c}$. On the other hand, if $0.5<\phi \leq 1$ for $\Omega_{d}$ and $0<\phi \leq 0.5$ for $\Omega_{c}$, then $H_{d}=\phi, H_{c}=1-\phi$, and $\phi=\max \left\{\phi_{1}, \ldots, \phi_{n_{d}}\right\}$ [12]. Further discussions on the regularization of Heaviside step function and Dirac delta function, as used in the context of the CLS method, are presented in [12].

\subsection{Mass transfer}

This research focuses on the simulation of external mass transfer from bubbles rising in a vertical channel. Therefore, a convection-diffusion-reaction equation is used as a mathematical model for the mass transfer of a chemical species in $\Omega_{c}$, as first introduced in [12]:

$$
\frac{\partial C}{\partial t}+\nabla \cdot(\mathbf{v} C)=\nabla \cdot(\mathcal{D} \nabla C)+\dot{r}(C),
$$

where $C$ is the chemical species concentration, $\mathcal{D}$ is the diffusion coefficient or diffusivity which is equal to $\mathcal{D}_{c}$ in $\Omega_{c}$ and $\mathcal{D}_{d}$ elsewhere, $\dot{r}(C)=-k_{1} C$ denotes the overall chemical reaction rate with first-order kinetics, and $k_{1}$ is the reaction rate constant. In the present model, the concentration inside the bubbles is kept constant [20|35|2|12], whereas convection, diffusion and reaction of the mass dissolved from $\Omega_{d}$ exists only in $\Omega_{c}$.

As introduced by [12], the concentration $\left(C_{P}\right)$ at the interface cells is computed by linear interpolation, using information of the concentration field from $\Omega_{c}$ (excluding interface cells), and taking into account that the concentration at the interface $C_{\Gamma}$ is constant. As a consequence, the concentration at the interface is imposed like a Dirichlet boundary condition, whereas Eq.(9) is computed in $\Omega_{c}$.

\subsection{Numerical methods}

The transport equations are solved with a finite-volume discretization on a collocated unstructured mesh, as introduced in [4]8]12]. For the sake of completeness, some points are reviewed in this manuscript. The convective term of momentum equation (Eq. (1)), CLS advection equation (Eq. (4)), and mass transfer equation for chemical species (Eq. (9)), is explicitly computed approximating the fluxes at cell faces with a Total Variation Diminishing (TVD) Superbee flux-limiter scheme proposed in [4|12]. Diffusive terms of transport equations are centrally differenced [12], whereas a distance-weighted linear interpolation is used to find the cell face values of physical properties and interface normals, unless otherwise stated. Gradients are computed at cell centroids by means of the least-squares method using information of the neighbor cells around the vertexes of the current cell (see Fig. 2 of [4]). For instance at the cell $\Omega_{P}$, the gradient of the variable $\psi=\left\{v_{j}, C, \phi_{i}, \phi_{i}\left(1-\phi_{i}\right), \ldots\right\}$ is calculated as follows:

$$
(\nabla \psi)_{P}=\left(\mathbf{M}^{T} \mathbf{W M}\right)^{-1} \mathbf{M}^{T} \mathbf{W} \mathbf{Y},
$$

$\mathbf{M}$ and $\mathbf{Y}$ are defined as introduced in [4], $\mathbf{W}=\operatorname{diag}\left(w_{P \rightarrow 1}, . ., w_{P \rightarrow n}\right)$ is the weighting matrix [28|31], defined as the diagonal matrix with elements $w_{P \rightarrow k}=\left\{1, \| \mathbf{x}_{P}-\right.$

ICCS Camera Ready Version 2019

To cite this paper please use the final published version:

$\begin{array}{lll}\text { DOI } & 10.1007 / 978-3-030-22747-0 \_45\end{array}$ 
$\left.\mathbf{x}_{k} \|^{-1}\right\}, k=\{1, . ., n\}$, and subindex $n$ is the number of neighbor cells. The impact of the selected weighting coefficient $\left(w_{P \rightarrow k}\right)$ on the simulations is evaluated in Section 3.1. The compressive term of the re-initialization equation (Eq. (5)) is discretized by a central-difference scheme [12]. The resolution of the velocity and pressure fields is achieved by using a fractional-step projection method [18]. In the first step a predictor velocity $\left(\mathbf{v}^{*}\right)$ is computed at cell-centroids, as follows:

$$
\frac{\rho \mathbf{v}^{*}-\rho^{n} \mathbf{v}^{n}}{\Delta t}=\mathbf{C}_{\mathbf{v}}^{n}+\mathbf{D}_{\mathbf{v}}^{n}+\left(\rho-\rho_{0}\right) \mathbf{g}+\sum_{i=1}^{n_{d}} \sigma \kappa_{i}\left(\phi_{i}\right) \nabla_{h} \phi_{i},
$$

where super-index $n$ denotes the previous time step, $\mathbf{D}_{\mathbf{v}}(\mathbf{v})=\nabla_{h} \cdot \mu \nabla_{h} \mathbf{v}+\nabla_{h}$. $\mu\left(\nabla_{h} \mathbf{v}\right)^{T}$, and $\mathbf{C}_{\mathbf{v}}(\rho \mathbf{v})=-\nabla_{h} \cdot(\rho \mathbf{v v})$. In a second step a corrected velocity (v) is computed at cell-centroids:

$$
\frac{\rho \mathbf{v}-\rho \mathbf{v}^{*}}{\Delta t}=-\nabla_{h}(p)
$$

Imposing the incompressibility constraint $\left(\nabla_{h} \cdot \mathbf{v}=0\right)$ to Eq. (12) leads to a Poisson equation for the pressure field at cells, which is computed by using a preconditioned conjugate gradient method:

$$
\nabla_{h} \cdot\left(\frac{1}{\rho} \nabla_{h} p\right)=\frac{1}{\Delta t} \nabla_{h} \cdot\left(\mathbf{v}^{*}\right),\left.\mathbf{e}_{\partial \Omega} \cdot \nabla_{h} p\right|_{\partial \Omega}=0 .
$$

Here, $\partial \Omega$ denotes the boundary of $\Omega$, excluding the periodic boundaries, where information of the corresponding periodic nodes is employed. Finally, to fullfill the incompressibility constraint, and to avoid the pressure-velocity decoupling on collocated meshes [34], a cell-face velocity $\mathbf{v}_{f}$ [48] is interpolated to advect momentum (Eq. (1)), CLS functions (Eq. (4)), and concentration (Eq. (9)), as explained in Appendix $\mathrm{B}$ of [8]. Temporal discretization of advection equation (Eq. (4)) and re-initialization equation (Eq. (5)) is done by using a TVD Runge-Kutta method [23]. Reinitialization equation (Eq. (5)), is solved for the steady state, using two iterations per physical time step [4]7[12].

Special attention is given to the discretization of convective (or compressive) term of transport equations. The convective term is approximated at $\Omega_{P}$ by $\left(\nabla_{h} \cdot \beta \psi \mathbf{c}\right)_{P}=$ $\frac{1}{V_{P}} \sum_{f} \beta_{f} \psi_{f} \mathbf{c}_{f} \cdot \mathbf{A}_{f}$, where $V_{P}$ is the volume of the current cell $\Omega_{P}$, subindex $f$ denotes the cell-faces, $\mathbf{A}_{f}=\left\|\mathbf{A}_{f}\right\| \hat{\mathbf{e}}_{f}$ is the area vector, $\mathbf{c}=\left\{\mathbf{v}, \mathbf{n}_{i}^{o}\right\}$, as introduced in [4]12]. Indeed, computation of variables $\psi=\left\{\phi_{i}, \phi_{i}\left(1-\phi_{i}\right), v_{j}, C, \ldots\right\}$ at the cell faces $\left(\psi_{f}\right)$ is performed as the sum of a diffusive upwind part $\left(\psi_{C_{p}}\right)$ plus an anti-diffusive term [4]8[12]:

$$
\psi_{f}=\psi_{C_{p}}+\frac{1}{2} L\left(\theta_{f}\right)\left(\psi_{D_{p}}-\psi_{C_{p}}\right) .
$$

where $L\left(\theta_{f}\right)$ is the flux limiter, $\theta_{f}=\left(\psi_{C_{p}}-\psi_{U_{p}}\right) /\left(\psi_{D_{p}}-\psi_{C_{p}}\right), C_{p}$ is the upwind point, $U_{p}$ is the far-upwind point, and $D_{p}$ is the downwind point [12]. Some of the fluxlimiters implemented on the unstructured multiphase solver [4|5|6|7|8|9|12], have the forms [40]:

$$
\begin{cases}\max \left\{0, \min \left\{2 \theta_{f}, 1\right\}, \min \left\{2, \theta_{f}\right\}\right\} & \text { Superbee } \\ 1 & \mathrm{CD}, \\ 0 & \text { Upwind. }\end{cases}
$$


Using TVD Superbee flux-limiter in the convective term of momentum equation benefits the numerical stability of the unstructured multiphase solver [4]5|6|7|8|9|12], especially for bubbly flows with high-density ratio and high Reynolds numbers, as demonstrated in our previous works [599]. Furthermore, $\left(\phi_{i}\right)_{f}$ in the convective term of Eq.(4) is computed using a Superbee flux-limiter (Eq.(15)). Nevertheless, other flux-limiters, e.g., TVD Van-Leer flux limiter, can be also employed as demonstrated in [12]. Regarding the variable $\left(\phi_{i}\left(1-\phi_{i}\right)\right)_{f}$ of the compressive term in Eq.(5), it can be computed by a central-difference limiter ( $\mathrm{CD}$ in Eq15), or equivalently by linear interpolation as detailed in Appendix A of [12]. The last approach is used in present simulations. The reader is referred to [4]5/6/8|9|12] for additional technical details on the finite-volume discretization of transport equations on collocated unstructured grids, which are beyond the scope of the present paper. Numerical methods are implemented in the framework of the parallel C++/MPI code TermoFluids [41]; whereas the parallel scalability of the multiple marker level-set solver is presented in [9]12].

\section{Numerical experiments}

Validations, applications and extensions of the unstructured CLS method [4] are reported in our previous works, for instance: dam-break problem [4], buoyancy-driven motion of single bubbles on unconfined domains [4677], binary droplet collision with bouncing outcome [5], drop collision against a fluid interface without coalescence [5], bubbly flows in vertical channels [9]11], falling droplets [10], energy budgets on the binary droplet collision with topological changes [1], Taylor bubbles [24|25], gas-liquid jets [38], thermocapillary migration of deformable droplets [7]13], and mass transfer from bubbles rising on unconfined domains [12]. Furthermore, a comparison of the unstructured CLS method [4] and coupled volume-of-fluid/level-set method [7] is reported in [10]. Therefore, this research can be considered as a further step for simulating mass transfer from buoyancy-driven bubbly flows in a wall confined vertical channel.

The hydrodynamics of bubbly flows in a vertical channel can be characterized by the following dimensionless numbers [17]:

$$
\begin{gathered}
M o=\frac{g \mu_{c}^{4} \Delta \rho}{\rho_{c}^{2} \sigma^{3}}, \quad E o=\frac{g d^{2} \Delta \rho}{\sigma}, \quad R e_{i}=\frac{\rho_{c} U_{T i} d}{\mu_{c}}, \\
\eta_{\rho}=\frac{\rho_{c}}{\rho_{d}}, \quad \eta_{\mu}=\frac{\mu_{c}}{\mu_{d}}, \quad C_{r}=\frac{D_{\Omega}}{d}, \quad \alpha=\frac{V_{d}}{V_{\Omega}},
\end{gathered}
$$

where, $\eta_{\rho}$ is the density ratio, $\eta_{\mu}$ is the viscosity ratio, $M o$ is the Morton number, Eo is the Eötvös number, $R e$ is the Reynolds number, $d$ is the initial bubble diameter, $\Delta \rho=\left|\rho_{c}-\rho_{d}\right|$ is the density difference between the fluid phases, subscript $d$ denotes the dispersed fluid phase, subscript $c$ denotes the continuous fluid phase, $\alpha$ is the bubble volume fraction, $C_{r}$ is the confinement ratio, $D_{\Omega}$ is the diameter of the circular channel, $V_{d}$ is the volume of bubbles $\left(\Omega_{d}\right), V_{\Omega}$ is the volume of $\Omega$, and $t^{*}=t \sqrt{g / d}$ is the dimensionless time. Numerical results will be reported in terms of the so-called drift velocity [22]12], $U_{T i}(t)=\left(\mathbf{v}_{i}(t)-\mathbf{v}_{\Omega}(t)\right) \cdot \hat{\mathbf{e}}_{y}$, which can be interpreted as the bubble velocity with respect to a stationary container, $\mathbf{v}_{i}(t)$ is the velocity of the ith bubble, $\mathbf{v}_{\Omega}(t)$ is the spatial averaged velocity in $\Omega$.

ICCS Camera Ready Version 2019

To cite this paper please use the final published version:

$\begin{array}{lll}\text { DOI } & 10.1007 / 978-3-030-22747-0 \_45\end{array}$ 

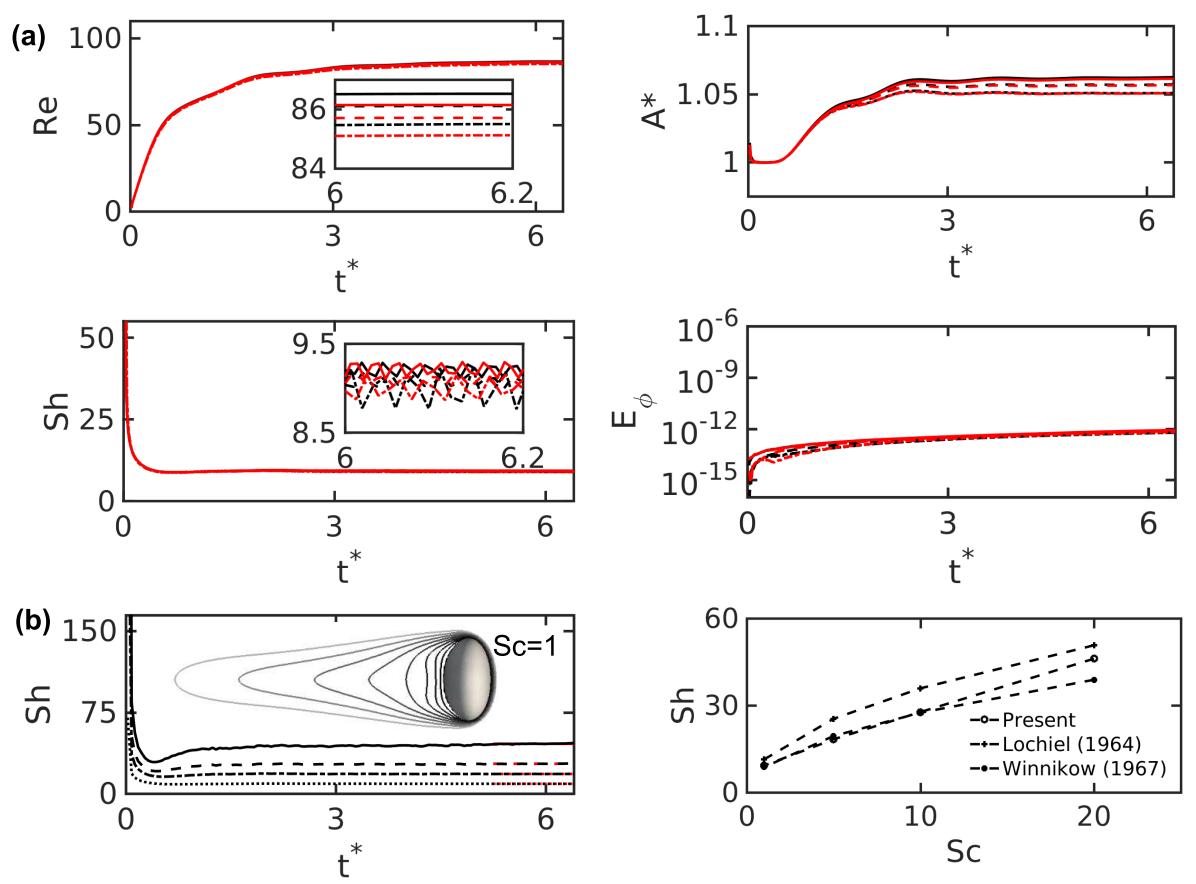

Fig. 1. Mass transfer from a single bubble, $E o=3.125, M o=1 \times 10^{-6}, \eta_{\rho}=\eta_{\mu}=100$, $S c=20, D a=0$ and $\alpha \approx 0 \%$. Grid size $h=\{d / 35(-), d / 30(--), d / 25(-\cdot)\}$. (a) Time evolution of Reynolds number $(R e)$, normalized surface of the bubble $\left(A^{*}(t)\right)$, Sherwood number $(S h(t))$, and mass conservation error $\left(E_{\phi}=\int_{\Omega}(\phi(\mathbf{x}, t)-\phi(\mathbf{x}, 0)) d V / \int_{\Omega} \phi(\mathbf{x}, 0) d V\right)$. Gradients evaluation (Eq. 101) with $w_{P \rightarrow k}=1$ (red lines) and $w_{P \rightarrow k}=\left\|\mathbf{x}_{P}-\mathbf{x}_{k}\right\|^{-1}$ (black lines). (b) Sherwood number for $S c=\{20(-), 10(--), 5(-\cdot), 1(\cdot)\}$ with a figure of mass concentration contours for $S c=1$, and comparison of present results against correlations [43]30].

Mass transfer with chemical reaction (first-order kinetics $\dot{r}(C)=-k_{1} C$ ) can be characterized by the Sherwood number $(S h)$, Schmidt number $(S c)$ or Peclet number $(P e)$, and the Damköler $(D a)$ number, defined in $\Omega_{c}$ as follows:

$$
S h=\frac{k_{c} d}{\mathcal{D}_{c}}, S c=\frac{\mu_{c}}{\rho_{c} \mathcal{D}_{c}}, P e=\frac{U_{T} d}{\mathcal{D}_{c}}=R e S c, D a=\frac{k_{1} d^{2}}{\mathcal{D}_{c}} .
$$

where $k_{c}$ is the mass transfer coefficient at the continuous fluid side.

\subsection{Validation and sensitivity to gradients evaluation}

In our previous work [12], extensive validation of the level-set model for mass transfer in bubbly flows has been presented. Here, the sensitivity of numerical simulations respect to gradients evaluation is researched, by simulating the mass transfer from a single buoyancy-driven bubble on an unconfined domain. $\Omega$ is a cylinder with height $H_{\Omega}=$ $10 d$ and diameter $D_{\Omega}=8 d$, where $d$ is the initial bubble diameter. $\Omega$ is discretized by

To cite this paper please use the final published version: 


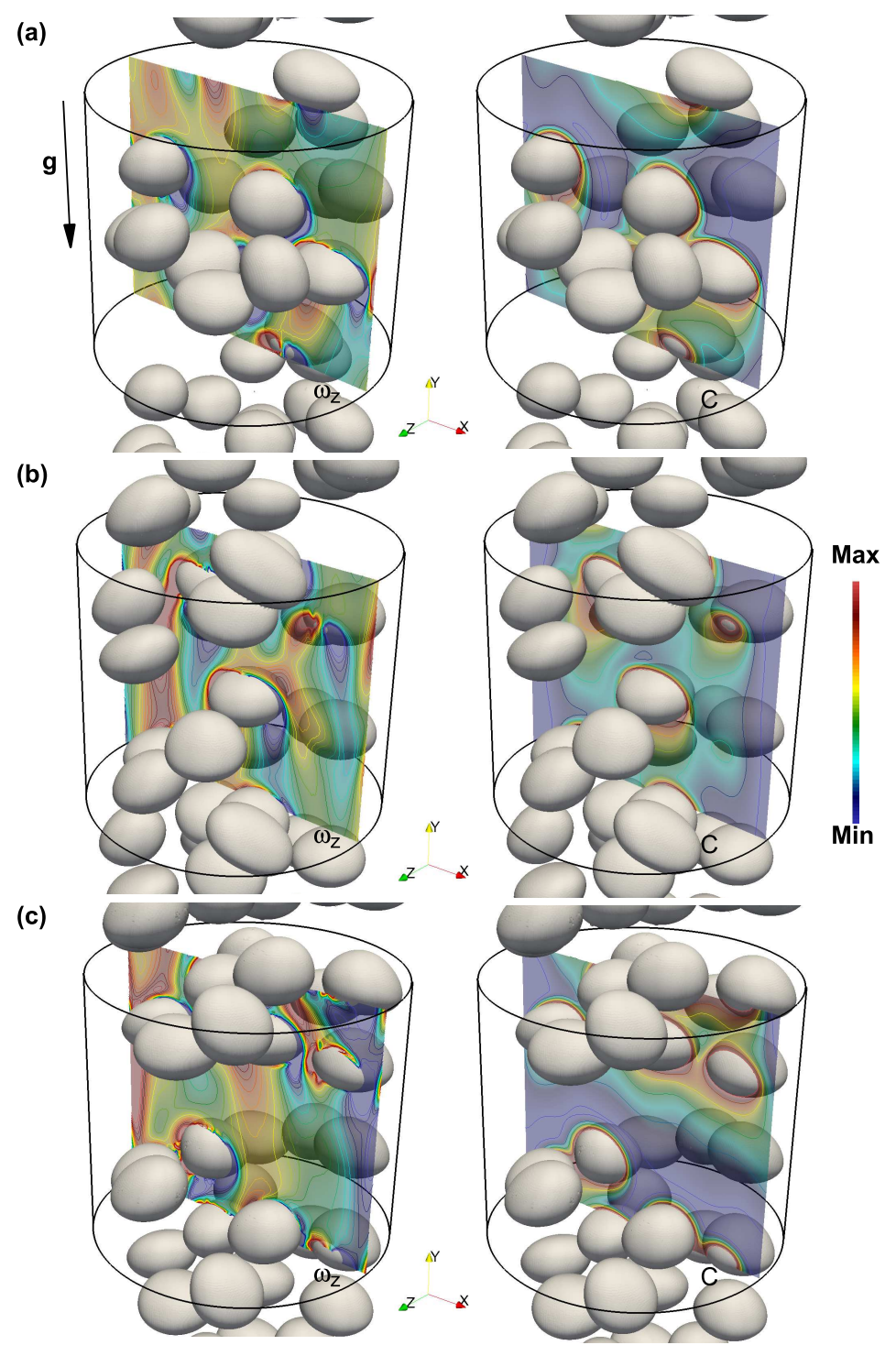

Fig. 2. Mass transfer from a bubble swarm (16 bubbles) in a periodic channel with circular crosssection, $E o=3.125, M o=5 \times 10^{-6}, \eta_{\rho}=\eta_{\mu}=100, S c=1, D a=7.97, \alpha=13.4 \%$. Vorticity $\left(\omega_{z}=\mathbf{e}_{z} \cdot \nabla \times \mathbf{v}\right)$ and concentration $(C)$ on the plane $x-y$ at (a) $t^{*}=t g^{1 / 2} d^{-1 / 2}=6.3$, (b) $t^{*}=12.5$, (c) $t^{*}=37.6$.

three unstructured meshes with $\left\{4.33 \times 10^{6}\left(M_{1}\right), 3.65 \times 10^{6}\left(M_{2}\right), 1.5 \times 10^{6}\left(M_{3}\right)\right\}$ triangular-prisms control volumes, distributed on $192 \mathrm{CPU}$-cores. Meshes are concentrated around the symmetry axis $y$, in order to maximize the bubble resolution, whereas the grid size in this region corresponds to $h=\left\{d / 35\left(M_{1}\right), d / 30\left(M_{2}\right), d / 25\left(M_{3}\right)\right\}$.

ICCS Camera Ready Version 2019

To cite this paper please use the final published version: 

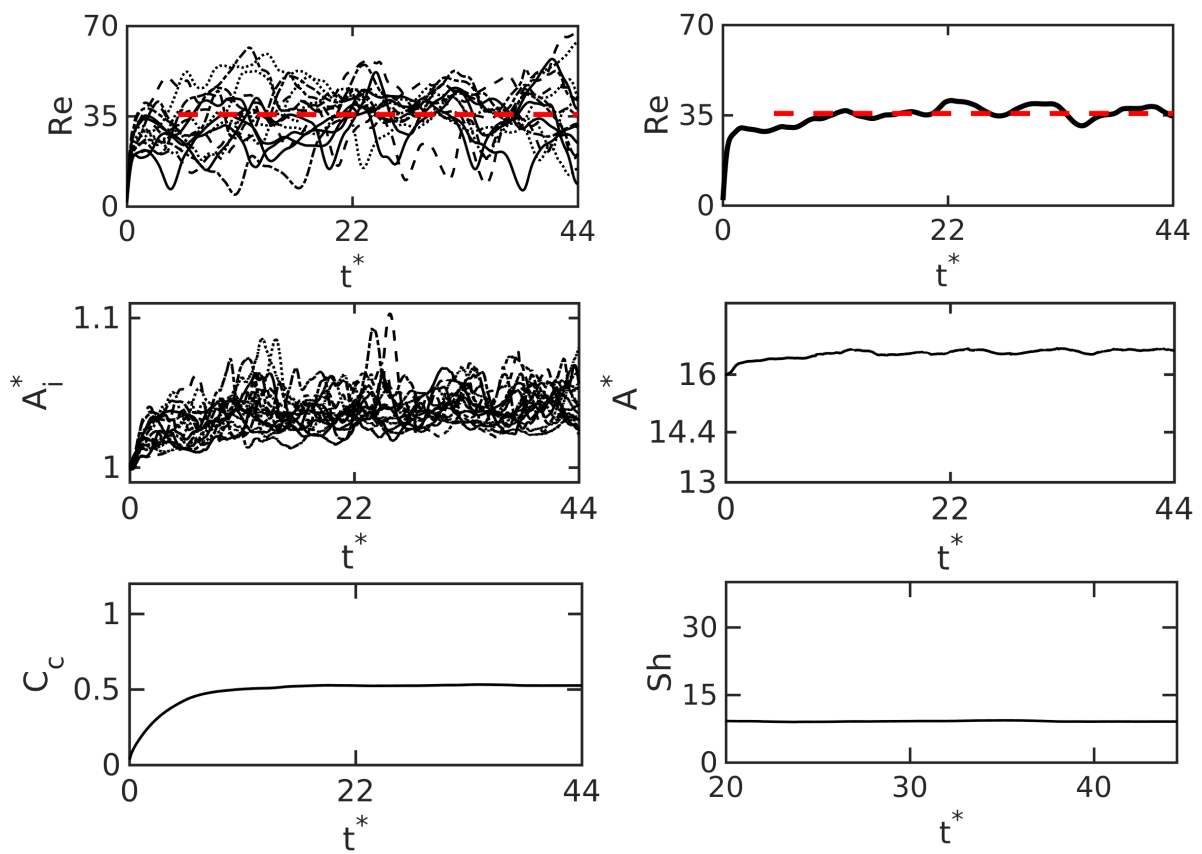

Fig. 3. Mass transfer from a bubble swarm ( 16 bubbles) in a periodic channel with circular crosssection, $E o=3.125, M o=5 \times 10^{-6}, \eta_{\rho}=\eta_{\mu}=100, S c=1, \alpha=11.8 \%$. Time evolution of Reynolds number ( $R e$ ) for each bubble (black lines), averaged Reynolds number (bold continuous line), time-averaged Reynolds number (red discontinuous line), normalized bubble surface $A_{i}^{*}(t)$, total interfacial surface of bubbles $A^{*}(t)=\sum_{i=1}^{n_{d}} A_{i}^{*}(t)$, spatial averaged concentration $C_{c}=V_{c}^{-1} \int_{\Omega_{c}} C d V$, and Sherwood number $S h(t)$.

Neumann boundary-condition is applied at lateral, top and bottom walls. The initial bubble position is $(x, y, z)=(0,1.5 d, 0)$, on the symmetry axis $y$, whereas both fluids are initially quiescent.

Mass transfer coefficient $\left(k_{c}\right)$ in single rising bubbles is calculated from a massbalance for the chemical species in $\Omega_{c}$, as follows [12]:

$$
k_{c}(t)=\frac{V_{c}}{A_{d}\left(C_{\Gamma, c}-C_{\infty}\right)} \frac{d C_{c}}{d t},
$$

where $C_{c}=V_{c}^{-1} \int_{\Omega_{c}} C(\mathbf{x}, t) d V, A_{d}=\int_{\Omega}\|\nabla \phi\| d V$ is the interfacial surface of the bubble, $V_{c}$ is the volume of $\Omega_{c}, C_{\Gamma, c}$ is the constant concentration on the bubble interface from the side of $\Omega_{c}$, and $C_{\infty}=0$ is the reference concentration. Dimensionless parameters are $E o=3.125, M o=1 \times 10^{-6}, D a=0, S c=\{1,5,10,20\}, \eta_{\rho}=100$ and $\eta_{\mu}=100$.

Fig 1. 1h shows the time evolution of Reynolds number $(R e)$, normalized interfacial surface $\left(A^{*}(t)\right)$, Sherwood number $(S h(t))$, and mass conservation error $\left(E_{\phi}\right)$, The grid-independence study shows that $h=d / 35$ is enough to perform accurate predictions of hydrodynamics and mass transfer from single bubbles. Furthermore, the effect 

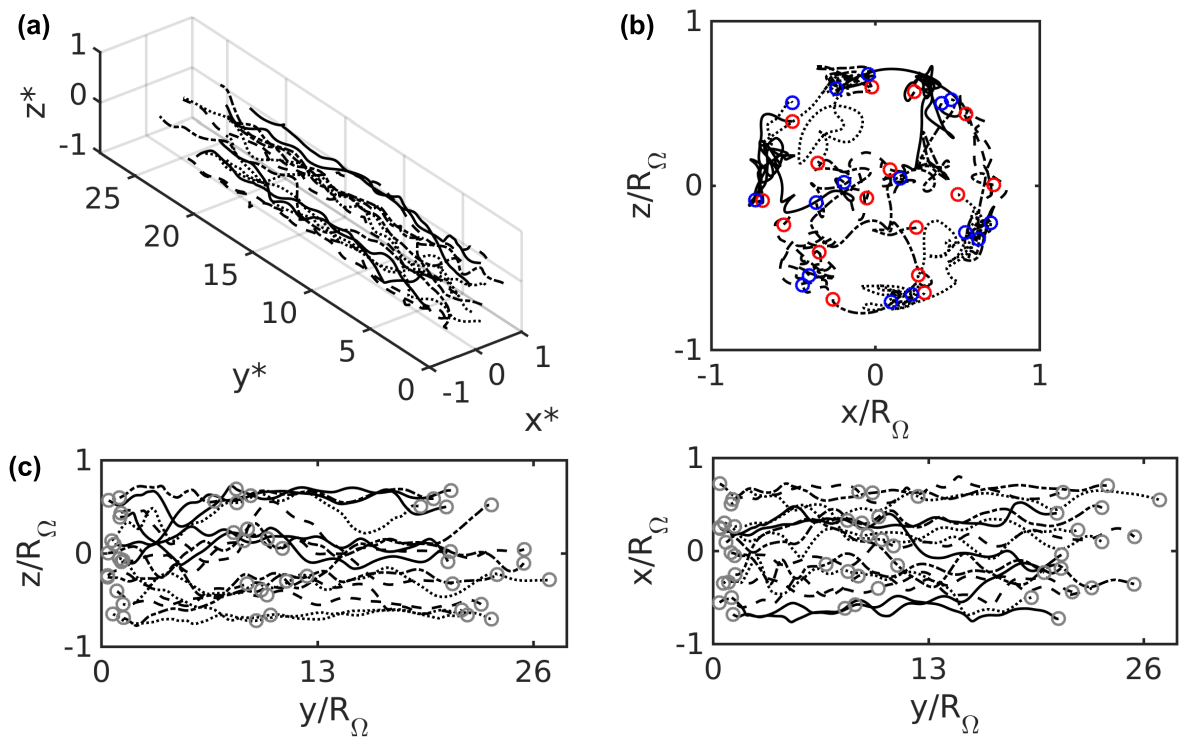

Fig. 4. Mass transfer from a bubble swarm (16 bubbles) in a periodic channel with circular crosssection, $E o=3.125, M o=5 \times 10^{-6}, \eta_{\rho}=\eta_{\mu}=100, S c=1, \alpha=11.8 \%$. (a) 3D bubble trajectories. (b) Projection of bubble trajectories on the plane $x-z$. (c) Projection of bubble trajectories on the plane $x-y$ and $z-y$. Here $R_{\Omega}=0.5 D_{\Omega}$ is the radius of the cylindrical channel, $\left(x^{*}, y^{*}, z^{*}\right)=\left(x / R_{\Omega}, y / R_{\Omega}, z / R_{\Omega}\right)$.

of gradients evaluation (Eq. (10) on the simulations, is depicted for weighting factors $w_{P \rightarrow k}=1$ (red lines) and $w_{P \rightarrow k}=\left\|\mathbf{x}_{P}-\mathbf{x}_{k}\right\|^{-1}$ (black lines). It is observed that numerical results are very close, whereas the numerical stability is maintained independently of the selected weighting factor. In what follows $w_{P \rightarrow k}=\left\|\mathbf{x}_{P}-\mathbf{x}_{k}\right\|^{-1}$ will be employed. Fig 1 b depicts the effect of Schmidt number on the Sherwood number, as well as a comparison of present results against empirical correlations from literature [43 30]. These results also give a further validation of the model for mass transfer coupled to hydrodynamics in buoyancy-driven bubbles.

\subsection{Mass transfer from a bubble swarm rising in a vertical channel}

As a further step and with the confidence that the CLS model has been validated [12], the mass transfer from a bubble swarm rising in a vertical pipe, is computed. The saturation of concentration in $\Omega_{c}$ is avoided by the chemical reaction term in Eq. (9] [35]12]. On the other hand, the mass transfer coefficient $\left(k_{c}\right)$ in $\Omega_{c}$ is computed by using a mass balance of the chemical species at steady state $\left(d C_{c} / d t=0\right)$, as follows [12]:

$$
k_{c}=\frac{V_{c} k_{1} C_{c}}{\left(C_{\Gamma, c}-C_{c}\right) \sum_{i=1}^{n d} A_{i}}
$$

ICCS Camera Ready Version 2019

To cite this paper please use the final published version: 
where $A_{i}=\int_{\Omega}\left\|\nabla \phi_{i}\right\| d V$ is the surface of the ith bubble, and $C_{c}=V_{c}^{-1} \int_{\Omega_{c}} C d V . \Omega$ is a periodic cylindrical channel, with height $H_{\Omega}=4 d$ and diameter $D_{\Omega}=4.45 d$, as depicted in Fig. $2 \Omega$ is discretized by $9.3 \times 10^{6}$ triangular-prisms control volumes, with grid size $h=d / 40$, distributed on 960 CPU-cores. Periodic boundary conditions are used on the $x-z$ boundary planes. On the wall, no-slip boundary condition for velocity, Dirichlet boundary condition for CLS markers $\left(\phi_{i}=1\right)$, and Neumann boundary condition for $C$. Bubbles are initially distributed in $\Omega$ following a random pattern, whereas fluids are quiescent. Since fluids are incompressible and bubble coalescence is not allowed, the void fraction $\left(\alpha=V_{d} / V_{\Omega}\right)$ and number of bubbles are constant throughout the simulation.

Dimensionless parameters are $E o=3.125, M o=5 \times 10^{-6}, S c=1, D a=7.97$, $\eta_{\rho}=100, \eta_{\mu}=100, \alpha=13.4 \%$ and $C_{r}=4.45$, which corresponds to a bubbly flow with 16 bubbles distributed in $\Omega$. Fig. 2 illustrates the mass transfer from a swarm of 16 bubbles at $t^{*}=\{6.26,37.6\}$. Concentration contours $(C)$, and vorticity contours $\left(\omega_{z}=\hat{\mathbf{e}}_{z} \cdot \nabla \times \mathbf{v}\right)$ are shown on the plane $x-y$. Fig. 3 depicts the time evolution of Reynolds number for each bubble and the time-averaged Reynolds number (discontinuous line), normalized bubble surface $A_{i}^{*}(t)$, total surface of bubbles $A^{*}(t)=\sum_{i=1}^{n_{d}} A_{i}^{*}(t)$, spatial averaged chemical species concentration $\left(C_{c}\right)$ in $\Omega_{c}$, and Sherwood number $S h(t)$ at steady state $(d C c / d t=0)$. Fig. 3 shows that $\operatorname{Re}_{i}(t)$ presents fluctuations, due to oscillations in the bubble shapes (see $A_{i}(t)$ ), and bubblebubble interactions such as bouncing interaction, and the so called drafting, kissing and tumbling processes [5|9[10]. On the other hand, the Reynold number of the bubble swarm, $\bar{R} e=n_{d}^{-1} \sum_{i=1}^{n_{d}} R e_{i}(t)$, achieves the steady-state. The spatial averaged concentration $\left(C_{c}\right)$ tend to the steady-state after a short transient, indicating an equilibrium between mass transfer from the bubbles and chemical reaction in $\Omega_{c}$. Furthermore, the mass transfer coefficient $(S h)$ achieves the steady-state, once $d C_{c} / d t=0$. Finally, Fig. 4 depict bubble trajectories, which indicate a bubble-wall repulsion effect.

\section{Conclusions}

DNS of mass transfer from buoyancy-driven bubbles rising in a vertical channel has been performed using a parallel multiple marker CLS method [5|9|12]. These numerical experiments demonstrate the capabilities of the present approach, as a reliable method for simulating bubbly flows with mass transfer and chemical reaction in vertical channels, taking into account bubble-bubble and bubble-wall interactions in long time simulation of bubbly flows. The method avoids the numerical merging of bubble interfaces, which is an issue inherent to standard interface capturing methods. Interactions of bubbles include a repulsion effect when these are horizontally aligned or when bubbles interact with the wall, whereas two bubbles vertically aligned tend to follow the so-called drafting-kissing-tumbling mechanism observed also in solid particles. These bubble-bubble and bubble-wall interactions lead to a fluctuating velocity field, analogous to that observed in turbulence. Nevertheless, the time averaged Reynolds number $(R e)$ and mass transfer coefficient $(S h)$ tend to the steady-state. Turbulence induced by agitation of bubbles promote the mixing of chemical species in the continuous phase, whereas the spatial averaged chemical species concentration tends to the 
steady-state, indicating a balance between chemical reaction in $\Omega_{c}$ and mass transfer from bubbles. These results demonstrate that the multiple marker CLS approach [12] is a predictive method to compute $S h=S h\left(E o, R e, D a, \alpha, C_{r}\right)$ in bubbly flows rising in a vertical channel. Future work includes the extension of this model to multicomponent mass transfer and complex chemical reaction kinetics, as well as parametric studies of $S h=S h\left(E o, R e, D a, \alpha, C_{r}\right)$ to develop closure relations for models based on the averaged flow (e.g., two-fluid models [27]).

\section{References}

1. Amani, A., Balcázar, N., Gutiérrez, E., Oliva, A., (2019). Numerical study of binary droplets collision in the main collision regimes. Chemical Engineering Journal 370, 477-498.

2. Aboulhasanzadeh, B., Thomas, S., Taeibi-Rahni, M., Tryggvason, G. (2012). Multiscale computations of mass transfer from buoyant bubbles. Chemical Engineering Science 75, 456-467.

3. Alke, A., Bothe, D., Kroeger, M., Warnecke, H.J. (2009). VOF-based simulation of conjugate mass transfer from freely moving fluid particles. In Mammoli, AA and Brebbia, CA (Ed): Computational methods in multiphase flow V, WIT Transactions on Engineering Sciences, 157-168.

4. Balcázar, N., Jofre, L., Lehmkhul, O., Castro, J., Rigola, J. (2014). A finite-volume/levelset method for simulating two-phase flows on unstructured grids. International Journal of Multiphase Flow 64, 55-72

5. Balcázar, N., Lehmkhul, O., Rigola, J., Oliva, A. (2015). A multiple marker level-set method for simulation of deformable fluid particles. International Journal of Multiphase Flow 74, 125-142

6. Balcázar, N., Lemhkuhl, O., Jofre, L., Oliva, A. (2015). Level-set simulations of buoyancydriven motion of single and multiple bubbles. International Journal of Heat and Fluid Flow 56, 91-107

7. Balcázar, N., Lehmkhul, O., Jofre, L., Rigola, J., Oliva, A. (2016). A coupled volume-offluid/level-set method for simulation of two-phase flows on unstructured meshes. Computers and Fluids 124, 12-29.

8. Balcázar, N., Rigola, J., Castro, J., Oliva, A. (2016). A level-set model for thermocapillary motion of deformable fluid particles. International Journal of Heat and Fluid Flow 62, Part B, 324-343.

9. Balcázar, N., Castro, J., Rigola, J., Oliva, A. (2017). DNS of the wall effect on the motion of bubble swarms. Procedia Computer Science 108, 2008-2017.

10. Balcázar, N., Castro, J., Chiva, J., Oliva, A. (2018). DNS of Falling Droplets in a Vertical Channel. International Journal of Computational Methods and Experimental Measurements, Volume 6 (2018), Issue 2., 398-410.

11. Balcázar N., Lehmkuhl O., Castro J., Oliva A. (2018). DNS of the Rising Motion of a Swarm of Bubbles in a Confined Vertical Channel. In: Grigoriadis D., Geurts B., Kuerten H., Fröhlich J., Armenio V. (eds) Direct and Large-Eddy Simulation X. ERCOFTAC Series, vol 24. Springer, Cham.

12. Balcázar-Arciniega N., Antepara O., Rigola J., Oliva, A., A level-set model for mass transfer in bubbly flows, International Journal of Heat and Mass Transfer, (Accepted, 2 Apr 2019), https://doi.org/10.1016/j.ijheatmasstransfer.2019.04.008

13. Balcázar N., Antepara O., Rigola J., Oliva A. DNS of Thermocapillary Migration of Deformable Droplets. In: Salvetti M., Armenio V., Fröhlich J., Geurts B., Kuerten H. (eds) Direct and Large-Eddy Simulation XI. ERCOFTAC Series, vol 25. Springer, Cham

ICCS Camera Ready Version 2019

To cite this paper please use the final published version:

$\begin{array}{lll}\text { DOI } & 10.1007 / 978-3-030-22747-0 \_45\end{array}$ 
14. Bothe, D., Koebe, M., Wielage, K., Warnecke, H.J. (2003). VOF simulations of mass transfer from single bubbles and bubble chains rising in the aqueous solutions, In Proceedings of FEDSM03: Fourth ASME-JSME Joint Fluids Engineering Conference, Honolulu, HI, USA, July 611.

15. Bothe, D., Fleckenstein, S. (2013). Modeling and VOF-based numerical simulation of mass transfer processes at fluidic particles. Chem. Eng. Science 101, 283-302.

16. Brackbill, J.U., Kothe, D.B., Zemach, C. (1992). A Continuum Method for Modeling Surface Tension, J. Comput. Phys. 100, 335-354.

17. Clift, R., Grace, J.R., Weber, M.E. Bubbles, Drops and Particles. Academin Press, New York, 1978.

18. Chorin, A.J. (1968). Numerical solution of the Navier-Stokes equations. Math. Comput. 22, 745-762.

19. Coyajee, E., Boersma, B.J., (2009). Numerical simulation of drop impact on a liquidliquid interface with a multiple marker front-capturing method. Journal of Computational Physics 228 (12), 4444-4467.

20. Darmana, D., Deen, N. G., Kuipers, J. A. M. (2006). Detailed 3D modeling of mass transfer processes in two-phase flows with dynamic interfaces, Chemical Engineering \& Technology 29(9), 1027-1033.

21. Davidson, M.R., Rudman, M. (2002). Volume-of-fluid calculation of heat or mass transfer across deforming interfaces in two-fluid flow. Numerical Heat Transfer, Part B: Fundamentals 41, 291-308.

22. Esmaeeli, A., Tryggvason, G. (1999). Direct numerical simulations of bubbly flows Part 2. Moderate Reynolds number arrays, J. Fluid Mech. 385, 325-358.

23. Gottlieb, S., Shu, C.W. (1998). Total Variation Dimishing Runge-Kutta Schemes, Mathematics of Computations 67, 73-85.

24. Gutiérrez, E., Balcázar, N., Bartrons, E., Rigola, J. (2017). Numerical study of Taylor bubbles rising in a stagnant liquid using a level-set/moving-mesh method. Chemical Engineering Science 164. 102-117.

25. Gutiérrez, E., Favre, F., Balcázar, N., Amani, A., Rigola, J., (2018). Numerical approach to study bubbles and drops evolving through complex geometries by using a level set Moving mesh Immersed boundary method. Chemical Engineering Journal 349. 662-682.

26. Hirt, C., Nichols, B. (1981). Volume of fluid (VOF) method for the dynamics of free boundary, J. Comput. Phys. 39, 201-225

27. Ishii, M., Hibiki, T., Thermo-Fluid Dynamics of Two-Phase Flow. 2nd edition, Springer, New-York, 2010.

28. Jasak, H. and Weller, H. G. (2000). Application of the finite volume method and unstructured meshes to linear elasticity, Int. J. Numer. Meth. Engng. 48, 267-287.

29. Koynov, A., Khinast, J. G., Tryggvason, G. (2005). Mass transfer and chemical reactions in bubble swarms with dynamic interfaces, AIChE Journal 51(10), 2786-2800.

30. Lochiel, A.,Calderbank,P. 1964. Mass transfer in the continuous phase around axisymmetric bodies of revolution. Chem.Eng.Sci. 19, 471-484.

31. Mavriplis, D. J., 2007. Unstructured Mesh Discretizations and Solvers for Computational Aerodynamics. 18th Computational Fluid Dynamics Conference, AIAA Paper 2007-3955, Miami, FL, doi:10.2514/6.2007-3955.

32. Olsson, E., Kreiss, G. (2005). A conservative level set method for two phase flow, J. Comput. Phys. 210, 225-246.

33. Osher, S., Sethian, J.A. (1988). Fronts propagating with curvature-dependent speed: Algorithms based on Hamilton-Jacobi formulations, J. Comput. Phys. 79, 175-210.

34. Rhie, C.M., Chow, W.L. (1983). Numerical Study of the Turbulent Flow past an Airfoil with Trailing Edge Separation, AIAA J. 21, 1525-1532.

ICCS Camera Ready Version 2019

To cite this paper please use the final published version:

$\begin{array}{lll}\text { DOI } & 10.1007 / 978-3-030-22747-0 \_45\end{array}$ 
35. Roghair, I., Van Sint Annaland, M., Kuipers, J.A.M. (2016). An improved Front-Tracking technique for the simulation of mass transfer in dense bubbly flows. Chem. Eng. Sci. 152, 351-369.

36. Sussman, M., Smereka, P., Osher, S. (1994). A Level Set Approach for Computing Solutions to Incompressible Two-Phase Flow, J. Comput. Phys. 144, 146-159.

37. Sussman, M., Puckett, E.G., 2000. A Coupled Level Set and Volume-of-Fluid Method for Computing 3D and Axisymmetric Incompressible Two-Phase Flows, J. Comput. Phys. 162, 301-337.

38. Schillaci, E., Antepara, O., Balcázar, N., Rigola, J., Oliva, A., 2019. A numerical study of liquid atomization regimes by means of conservative level-set simulations, Computers \& Fluids $179,137-149$.

39. Sun, D.L., Tao, J., W.Q., 2010. A coupled volume-of-fluid and level-set (VOSET) method for computing incompressible two-phase flows, International Journal of Heat and Mass Transfer 53, 645-655.

40. Sweby, P.K., 1984. High Resolution Using Flux Limiters for Hyperbolic Conservation Laws, SIAM Journal on Numerical Analysis 21, 995-1011.

41. Termo Fluids S.L., http: / / www . termofluids . com/ Last accessed 29 January 2019.

42. Tryggvason, G., Bunner, B., Esmaeeli, A., Juric, D., Al-Rawahi, N., Tauber, W., Han, J., Nas, S., Jan, Y-J. (2001). A Front-Tracking Method for the Computations of Multiphase Flow, $J$. Comput. Phys. 169, 708-759.

43. Winnikow, S., 1967. Letter to the Editors. Chem. Eng. Sci. 22 (3), 477.

ICCS Camera Ready Version 2019

To cite this paper please use the final published version:

\begin{tabular}{l|l|} 
DOI & $10.1007 / 978-3-030-22747-0 \_45$
\end{tabular} 